UCB-PTH-99/13

LBNL-43085

hep-ph/9904236

April 1999

\title{
Electroweak Symmetry Breaking and Large Extra Dimensions
}

\author{
Lawrence Hall and Christopher Kolda \\ Department of Physics \\ University of California \\ Berkeley, CA 94530, USA \\ and \\ Theory Group \\ Lawrence Berkeley National Laboratory \\ Berkeley, CA 94530, USA
}

\begin{abstract}
If spacetime contains large compact extra dimensions, the fundamental mass scale of nature, $\Lambda$, may be close to the weak scale, allowing gravitational physics to significantly modify electroweak symmetry breaking. Operators of the form $\left(1 / \Lambda^{2}\right)\left|\varphi^{\dagger} D^{\mu} \varphi\right|^{2}$ and $\left(1 / \Lambda^{2}\right) \varphi^{\dagger} W_{\mu \nu} B^{\mu \nu} \varphi$, where $W_{\mu \nu}$ and $B^{\mu \nu}$ are the $S U(2)$ and $U(1)$ field strengths and $\varphi$ is the Higgs field, remove the precision electroweak bound on the Higgs boson mass for values of $\Lambda$ in a wide range: $4 \mathrm{TeV} \lesssim \Lambda \lesssim 11 \mathrm{TeV}$. Within this framework, there is no preference between a light Higgs boson, a heavy Higgs boson, or a non-linearly realized $S U(2) \times U(1)$ symmetry beneath $\Lambda$. If there is a Higgs doublet, then operators of the form $\left(1 / \Lambda^{2}\right) \varphi^{\dagger} \varphi\left(G^{2}, F^{2}\right)$, where $G_{\mu \nu}$ and $F_{\mu \nu}$ are the QCD and electromagnetic field strengths, modify the production of the Higgs boson by gluon-gluon fusion, and the decay of the Higgs boson to $\gamma \gamma$, respectively. At Run II of the Tevatron collider, a $\gamma \gamma$ signal for extra dimensions will be discovered if $\Lambda$ is below 2.5 (1) TeV for a Higgs boson of mass 100 (300) GeV. Furthermore, such a signal would point to gravitational physics, rather than to new conventional gauge theories at $\Lambda$. The discovery potential of the LHC depends sensitively on whether the gravitational amplitudes interfere constructively or destructively with the standard model amplitudes, and ranges from $\Lambda=3-10(2-4)$ $\mathrm{TeV}$ for a light (heavy) Higgs boson.
\end{abstract}




\section{Introduction}

The conventional framework for particle physics beyond the standard model (SM) assumes that the fundamental mass scale of nature is the Planck mass: $M_{P l} \approx 10^{19} \mathrm{GeV}$. It is then natural to ask: why are the masses of the elementary particles so small? Proposed solutions to this hierarchy problem have a common feature: new non-perturbative gauge interactions dynamically generate a much lower scale, $M_{d y n}$, from which electroweak symmetry breaking is generated, and hence all the masses of the known elementary particles. Schematically, this mass hierarchy is

$$
M_{P l} \rightarrow M_{d y n} \rightarrow M_{W} \ldots m_{e}
$$

In supersymmetric theories, $M_{d y n}$ is the scale at which supersymmetry is broken, and the triggering of electroweak symmetry breaking may be mediated, for example, by gravitationalscale physics, or by gauge interactions at much lower energy scales. Alternatively, $M_{d y n}$ may be the scale of a new gauge force, technicolor, which forms fermion condensates that directly break $S U(2) \times U(1)$. Finally, new strong gauge forces could bind a composite Higgs boson.

Recently an alternative framework has been proposed [1] in which spacetime is enlarged to contain large extra compact spatial dimensions. At distances smaller than the size of these extra dimensions the gravitational force varies more rapidly than the inverse square law, so that the fundamental mass scale of gravity can be made much smaller than $M_{P l}$. The conventional mass hierarchy of (1) is completely avoided if this fundamental mass scale is of order the weak scale. In this case, the length scale of the extra dimensions is much larger than the scales probed experimentally at colliders, and hence this framework requires that the quarks, leptons and gauge quanta of the SM are spatially confined to a $3+1$ dimensional sub-space of the enlarged spacetime.

The physics at the fundamental scale, $\Lambda$, which may well be that of string theory, will be directly accessible to colliders of sufficiently high energy; but even at lower energies this physics may be experimentally probed. At energies below the fundamental mass scale, physics is described by an effective Lagrangian, which we take to be the most general set of $S U(3) \times S U(2) \times U(1)$ invariant operators involving quark, lepton and Higgs doublet fields of the SM:

$$
\mathcal{L}_{e f f}=\mathcal{L}_{S M}+\sum_{i} \frac{c_{i}}{\Lambda^{p}} \mathcal{O}_{i}^{4+p}
$$

where $\mathcal{L}_{S M}$ is the SM Lagrangian, $i$ runs over all gauge invariant operators, $\mathcal{O}_{i}^{4+p}$, of dimension $4+p$ with $p \geq 1$, and $c_{i}$ are unknown dimensionless couplings.

In this letter we study consequences of several of the dimension-6 operators. First we derive bounds on the $c_{i} / \Lambda^{2}$ from existing experimental results under very conservative assumptions about flavor-breaking in the ultraviolet theory. We then re-examine the precision electroweak bounds on the Higgs boson mass. Analyses within the standard model find a light Higgs; however, we will show that such results do not survive the addition of non-renormalizable operators, even if those operators are suppressed by scales as large as $11 \mathrm{TeV}$. In theories with large extra dimensions there is no good argument for a light Higgs over a heavy Higgs or a non-linearly realized $S U(2) \times U(1)$ symmetry, in which case (2) must be replaced by a chiral Lagrangian. Finally we examine two operators in particular 
and their effects on the discovery of Higgs bosons:

$$
\begin{aligned}
\mathcal{O}_{G} & =\varphi^{\dagger} \varphi G_{\mu \nu}^{a} G^{a \mu \nu} \\
\mathcal{O}_{\gamma} & =\varphi^{\dagger} \varphi F_{\mu \nu} F^{\mu \nu}
\end{aligned}
$$

where $G_{\mu \nu}^{a}$ and $F^{\mu \nu}$ are the QCD and electromagnetic field strengths, and $\varphi$ is the Higgs doublet with $\operatorname{Re} \varphi^{0}=(v+h) / \sqrt{2}$. The first operator contributes to Higgs production at hadron colliders via gluon-gluon fusion, and the second to Higgs decay to $\gamma \gamma$. There are two reasons why these effects provide a significant discovery potential for extra dimensions: first, they are competing against a SM signal which is suppressed by loop factors, and second, the $\operatorname{SM} \Gamma(h \rightarrow \gamma \gamma)$ is further suppressed by $e^{4} \simeq 10^{-2}$, where $e$ is the electromagnetic coupling constant.

However we assume that the physics at scale $\Lambda$ which generates (3)-(4), does so in a way that the coefficients are not suppressed by powers of the SM gauge coupling constants (see also [2]). Such a behavior is certainly not expected if the theory at $\Lambda$ is a 4-dimensional gauge field theory: in that case operators of the form (3)-(画) would arise by integrating out heavy fields, but these fields must couple to $F_{\mu \nu}$ and $G_{\mu \nu}$ with the usual SM gauge couplings, and further, as shown in [3], they must be also be loop-suppressed. Thus even if the gauge theory at $\Lambda$ were strongly-coupled, it seems unlikely that coefficients of $\mathcal{O}(1)$ could be generated. This is very important - the effect of the interaction $\left(e^{2} / \Lambda^{2}\right) \varphi^{\dagger} \varphi F^{2}$ on the $h \rightarrow \gamma \gamma$ branching ratio has been studied, and is small for $\Lambda \geq 1 \mathrm{TeV}$ 件. Thus observation of the physics we will describe in Section 5 would provide support for an extra-dimensional theory.

\section{Some Constraints on $\Lambda$}

Are the coefficients $c_{G, \gamma} / \Lambda^{2}$ expected to be large enough for an observable $h \rightarrow \gamma \gamma$ signal? In general this cannot be excluded, since physics induced by operators $\mathcal{O}_{i}$ will place bounds on

$$
\frac{f_{i}}{\Lambda_{i}^{p}} \equiv \frac{c_{i}}{\Lambda^{p}} \quad\left(f_{i}= \pm 1\right)
$$

not on $\Lambda$. However, it would be unreasonable to expect $c_{G, \gamma}$ to be orders of magnitude larger than all the other $c_{i}$.

It is tempting to assume that although the dimensionless coefficients $c_{i}$ are unknown, they are all of order unity. However, in this case operators which violate baryon number constrain $\Lambda \gtrsim 10^{16} \mathrm{GeV}$, and $C P$ violating operators contributing to $\epsilon_{K}$ constrain $\Lambda \gtrsim 10^{5} \mathrm{GeV}$. Thus the framework of large compact extra dimensions, allowing a fundamental scale close to the weak scale, is clearly excluded unless the low energy effective theory possesses an approximate flavor symmetry, in which case one expects

$$
c_{i}=\varepsilon_{F i} c_{i}^{\prime}
$$

with $c_{i}^{\prime}$ of order unity. The flavor symmetry breaking parameters, $\varepsilon_{F i}$, depend on the flavor symmetry group and the pattern of flavor symmetry breaking. For operators which violate flavor and $C P$ they must be small, while for operators which conserve flavor and $C P$ they may be set to unity. 
To allow low values for $\Lambda$, the flavor group should be large, and its breaking should be kept to a minimum, consistent with the observed quark and lepton masses and mixings. The maximum flavor group of the SM is $U(3)^{5}$. The three generations of quarks and leptons transform as $q_{L}=\left(u_{L}, d_{L}\right) \sim(3,1,1,1,1) ; u_{R} \sim(1,3,1,1,1) ; d_{R} \sim(1,1,3,1,1) ; \ell_{L}=$ $\left(\nu_{L}, e_{L}\right) \sim(1,1,1,3,1) ; e_{R} \sim(1,1,1,1,3)$. If there are only three symmetry breaking parameters, one for each of the up, down and charged lepton mass matrices, $\varepsilon_{u} \sim(3, \overline{3}, 1,1,1)$; $\varepsilon_{d} \sim(3,1, \overline{3}, 1,1) ; \varepsilon_{e} \sim(1,1,1,3, \overline{3})$, then baryon number and lepton number remain unbroken. (The $\varepsilon_{i}$ are equal to the Yukawa couplings up to an $\mathcal{O}(1)$ factor, $c_{i}: \lambda_{u, d, e}=c_{u, d, e} \varepsilon_{u, d, e}$.) However, even after imposing such a flavor symmetry, there remain operators such as

$$
\mathcal{O}_{q q}=\left(\bar{q}_{L} \gamma^{\mu} \varepsilon_{u} \varepsilon_{u}^{\dagger} q_{L}\right)^{2}=c_{u}^{4}\left(\bar{q}_{L} \gamma^{\mu} \lambda_{u} \lambda_{u}^{\dagger} q_{L}\right)^{2}
$$

which contribute to $\epsilon_{K}$ and constrain $\Lambda \gtrsim 4.2 \mathrm{TeV} \times\left(\sqrt{c_{q q}} / c_{u}^{2}\right)$. There are two ways to avoid this bound. First, since the bound depends quadratically on $c_{u}$, values slightly larger than 1 will weaken the bound significantly; this seems entirely natural to us. Second, one could postulate that $\varepsilon_{u, d}$ are real and the observed $\epsilon_{K}$ has an exotic origin; we view this as disfavored given that measurements of $V_{u b} / V_{c b}$ and $B-\bar{B}$ mixing indicate values of the CKM matrix elements consistent with a standard model origin of $\epsilon_{K}$ to better than $30 \%$.

For the $h \rightarrow \gamma \gamma$ signal, we are interested in the operators (3)-(四), which conserve $U(3)^{5}$. Hence, even if the higher dimension flavor violating operators, such as (7), are completely absent, it is important to study constraints on $\Lambda$ expected from operators which conserve $U(3)^{5}$. Such operators include flavor-conserving four-fermion operators and operators involving the Higgs doublet and the gauge fields. There have been many analyses to date which obtain constraints from these operators, and here we will simply repeat the results of these analyses, in the notation we are using for $\Lambda$. (An analysis similar to ours was recently presented in [2].)

Among the $C P$-conserving four-fermion operators, the strongest constraints come from atomic parity violation. The operator

$$
\mathcal{O}_{\ell q}=\left(\bar{\ell}_{L} \gamma_{\mu} \ell_{L}\right)\left(\bar{q}_{L} \gamma^{\mu} q_{L}\right)
$$

gives a constraint $\Lambda_{l q}>3.0 \mathrm{TeV}$ [5] at $95 \% \mathrm{CL}$. If the operator $\left(\bar{e}_{R} \gamma_{\mu} e_{R}\right)\left(\bar{q}_{L} \gamma^{\mu} q_{L}\right)$ were generated with the same coefficient, $P$ would be preserved in atomic systems and the previous limit would vanish. Although we do not expect $P$ to be a good symmetry of the underlying theory, a partial cancellation could easily weaken this bound. Apart from $P$-violation, the best bounds on $\Lambda_{\ell q}$ currently come from OPAL [6], using the $\ell_{1}, q_{3}$ component, and from CDF [7], using the $\ell_{2}, q_{1}$ component. Both find $\Lambda>800 \mathrm{GeV}$ at $95 \% \mathrm{CL}$.

The bounds on the coefficients of the operators $\mathcal{O}_{q q, \ell q}$ of (7)-(8) do not provide strict bounds on the scale $\Lambda$, because $\Lambda=\Lambda_{i} \sqrt{c_{i}}$, and the $c_{i}$ are unknown. Nevertheless, if the (flavor-conserving) $c_{i}^{\prime}=c_{i}$ are expected to be of order unity for these operators, then $\Lambda \gtrsim 3 \mathrm{TeV}$ is clearly allowed, while a value of $\Lambda$ as low as $1 \mathrm{TeV}$ seems disfavored.

\section{Precision Electroweak Physics and the Higgs Mass Bound}

A second class of constraints arise from precision measurements in the electroweak gauge sector, namely from the $S$ and $T$ parameters (see, e.g., [\&]). The strongest of these con- 
straints arise from the operators:

$$
\begin{aligned}
\mathcal{O}_{B W} & =B^{\mu \nu}\left(\varphi^{\dagger} \tau^{a} W^{a \mu \nu} \varphi\right) \\
\mathcal{O}_{\Phi} & =\left(\varphi^{\dagger} D^{\mu} \varphi\right)\left(D_{\mu} \varphi^{\dagger} \varphi\right)
\end{aligned}
$$

which contribute

$$
\begin{aligned}
\Delta S_{\text {new }} & =-\frac{2 c_{W} s_{W}}{\alpha} \frac{v^{2}}{\Lambda_{B W}^{2}} f_{B W} \\
\Delta T_{\text {new }} & =-\frac{1}{2 \alpha} \frac{v^{2}}{\Lambda_{\Phi}^{2}} f_{\Phi}
\end{aligned}
$$

where $s_{W}, c_{W}$ are the sine and cosine of the weak angle and $f_{B W}, f_{\Phi}$ are unknown signs.

A global fit to electroweak observables [9] yields $S_{f i t}=-0.14 \pm 0.12$ and $T_{\text {fit }}=-0.22 \pm$ 0.15 assuming $m_{h}=100 \mathrm{GeV}$. I Since each operator contributes only to one of $S$ or $T$, we can find independent bounds on each. We find that at 95\% CL:

$$
\begin{aligned}
\Lambda_{B W} & >3.6 \mathrm{TeV} \\
\Lambda_{\Phi} & >3.0 \mathrm{TeV} .
\end{aligned}
$$

We can also extract a bound if $\Lambda_{B W}=\Lambda_{\Phi}: \Lambda>4.0 \mathrm{TeV}$, allowing the Higgs mass to vary over the range $100 \mathrm{GeV}<m_{h}<800 \mathrm{GeV}$. We see that the constraints from precision electroweak physics are very similar in magnitude to those obtained in the previous section.

How important are these constraints for restricting $\Lambda_{\gamma}$ ? Although the electromagnetic field strength, $F^{\mu \nu}$, is not $S U(2) \times U(1)$ invariant, the operator $\mathcal{O}_{\gamma}$ is generated, after electroweak symmetry-breaking, from the invariant operators $\mathcal{O}_{B}=\left(\varphi^{\dagger} \varphi\right) B_{\mu \nu} B^{\mu \nu}, \mathcal{O}_{W}=$ $\left(\varphi^{\dagger} \varphi\right) W_{\mu \nu} W^{\mu \nu}$ and $\mathcal{O}_{B W}$ of Eq. (9):

$$
\frac{f_{\gamma}}{\Lambda_{\gamma}^{2}}=c_{W}^{2} \frac{f_{B}}{\Lambda_{B}^{2}}+s_{W}^{2} \frac{f_{W}}{\Lambda_{W}^{2}}+c_{W} s_{W} \frac{f_{B W}}{\Lambda_{B W}^{2}}
$$

If all $f_{i}$ and $\Lambda_{i}$ on the right side of Eq. (15) were equal, then the bound (13) on $\Lambda_{B W}$ implies $\Lambda_{\gamma}>3.3 \mathrm{TeV}$. However, changes in the relative signs or sizes of each contribution significantly reduces the bound; thus we have no strong lower bound on the scale $\Lambda_{\gamma}$ itself. Likewise we know of no strong constraint on the scale $\Lambda_{G}$ either.

Finally we wish to address the question of the Higgs mass. It is well-known that fits to the electroweak data indicate a light Higgs. A simple fit can be done using only $S$ and $T$ as given above and the following parameterization of the Higgs contributions from Ref. [8]:

$$
\begin{aligned}
\Delta S_{H} & =0.091 x_{H}-0.010 x_{H}^{2} \\
\Delta T_{H} & =-0.079 x_{H}-0.028 x_{H}^{2}+0.0026 x_{H}^{3}
\end{aligned}
$$

where $x_{H}=\log \left(m_{h} / 100 \mathrm{GeV}\right)$. Using these forms, one can do a fit demanding $S_{f i t}=$ $\Delta S_{H}+\Delta S_{\text {new }}$ and likewise for $T$. For the SM alone, a 95\% CL upper bound of $255 \mathrm{GeV}$ has

\footnotetext{
${ }^{1}$ The fit in [9] uses $m_{h}=600 \mathrm{GeV}$ and defines $S=T=0$ in the SM. We rescale to $m_{h}=100 \mathrm{GeV}$ using the parameterization of Ref. [8] (see Eqs. (16)-(17)). We then treat deviations from $m_{h}=100 \mathrm{GeV}$ as "new physics."
} 
been obtained [9]. However it is clear that from the point of view of the oblique parameters, shifts in $\Delta S_{H}$ and $\Delta T_{H}$ can be compensated by similar shifts in $\Delta S_{\text {new }}$ and $\Delta T_{\text {new }}$. Thus we can derive an effective "95\% CL bound" on the Higgs mass as a function of $\Lambda$ under the requirement that the fit to the experimentally obtained $S_{f i t}$ and $T_{f i t}$ be no worse than that obtained for $m_{h}=255 \mathrm{GeV}$ and $\Lambda \rightarrow \infty$. (We do this by constructing a $\chi^{2}$ distribution from $S$ and $T$ alone.)

How large can the Higgs mass become with the inclusion of $\mathcal{O}_{B W}$ and $\mathcal{O}_{\Phi}$ ? The answer is: quite large. Fitting to $m_{h}$ as a function of $\Lambda$ and using $S$ and $T$ as "experimental" inputs, we find for particular choices of the signs of the operators (i.e., $f_{B W}=f_{\Phi}=+1$ ) that the precision electroweak bound on the Higgs mass disappears completely for $4 \mathrm{TeV} \lesssim \Lambda \lesssim 11 \mathrm{TeV}$ ! (By "disappear" we mean that the $95 \%$ upper bound on $m_{h}$ exceeds the unitarity bound of approximately $800 \mathrm{GeV}$ and so is meaningless.) Thus, in the context of gravitational physics at or below $10 \mathrm{TeV}$, the usual claims that electroweak physics prefers a light Higgs do not hold. And even for $\Lambda$ as high as $17 \mathrm{TeV}$, the upper limit on the Higgs mass exceeds $500 \mathrm{GeV}$.

These results are summarized in Figure 1 where we show the 95\% CL allowed range for $m_{h}$ as a function of $\Lambda \equiv \Lambda_{B W}=\Lambda_{\Phi}$. The hatched region at small $\Lambda$ is ruled out because of its large contribution to $S$ and $T$, while the region at large $\Lambda$ and large $m_{h}$ is ruled out because the new operators contribute too little to $S$ and $T$ to significantly effect the SM fit to the Higgs mass. However for intermediate $\Lambda$ (unhatched region) it is clear that there is effectively no limit on the Higgs mass thanks to the effects of the new operators.

(If the physics at $\Lambda$ were weakly-coupled then we would expect that $c_{B W} \simeq e^{2} c_{W} s_{W}$; then allowing $c_{\Phi} \simeq 1 / 4$ would reproduce Fig. 1, only with the $\Lambda$ rescaled by $\sim 1 / 2$. Thus the preference for a light Higgs in the SM is even removed for a weakly-coupled gauge theory if $\Lambda \sim 2-5 \mathrm{TeV}$.)

Finally, we note that the one other argument for a light Higgs, namely triviality, is no longer applicable in these models either. With such a low ultraviolet cutoff $(\Lambda \sim$ few TeV $)$, the Higgs self-coupling cannot run to its Landau pole for $m_{h} \lesssim 1 \mathrm{TeV}$.

\section{Implications for Electroweak Symmetry Breaking}

The mechanism for electroweak symmetry breaking (EWSB) is unknown. Nevertheless, it is commonly believed that the Higgs boson exists, and is light. The two indirect indications for this are:

- The successful prediction of the weak mixing angle from gauge coupling constant unification. This prediction results in theories with weak scale supersymmetry which are perturbative to a high scale; such theories have a light Higgs boson, $m_{h} \lesssim 150 \mathrm{GeV}$ [10].

- The experimental values of the precision electroweak observables are consistent with the standard model, at 95\% C.L., only if $m_{h} \lesssim 255 \mathrm{GeV}$ 9].

If there are large extra dimensions allowing the fundamental scale, $\Lambda$, to be in the $\mathrm{TeV}$ domain, neither of these points can be used to argue that the Higgs boson is light. For the first: it has not been demonstrated that it is possible to predict the weak mixing angle to the percent level of accuracy in these theories; furthermore, there is no need for the field 


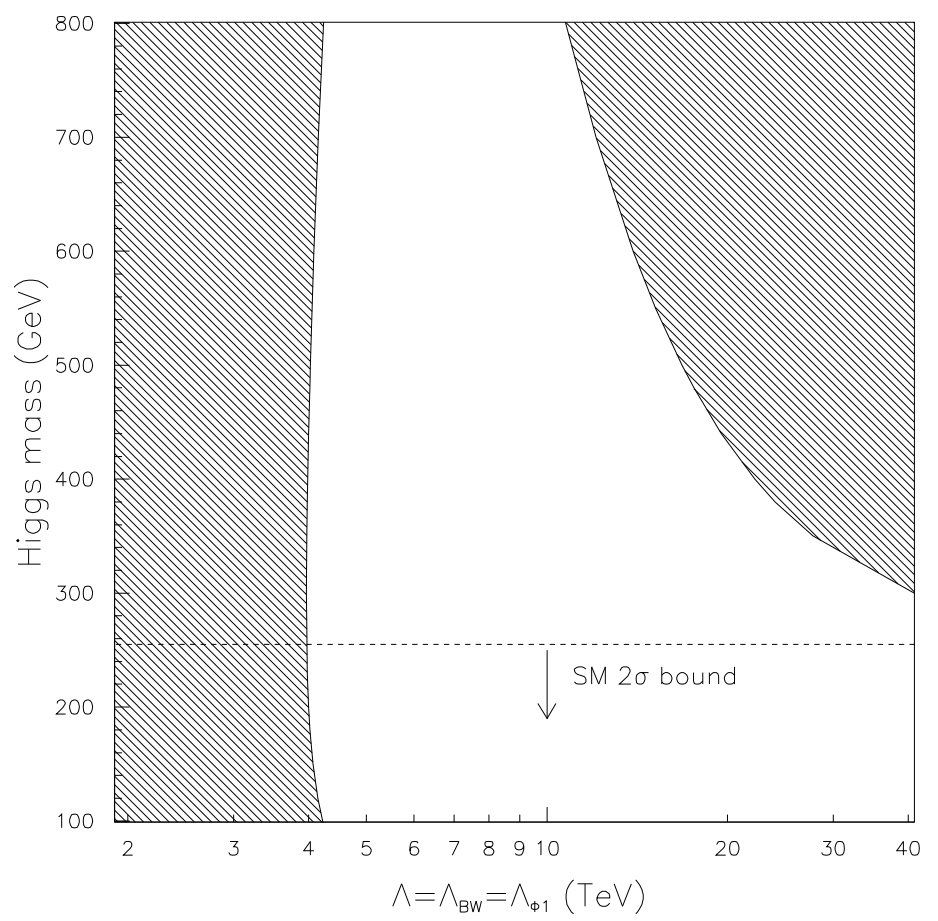

Figure 1: Precision electroweak limits on the Higgs mass as a function of the scale of new physics. For this figure, $\Lambda_{B W}$ and $\Lambda_{\Phi}$ are chosen equal, while the signs $f_{B W}$ and $f_{\Phi}$ are chosen to maximize the allowed region. Hatched regions are disallowed at $95 \%$, while the dashed line borders the region allowed in the SM alone.

theory below $\Lambda$ to be supersymmetric since there is no large hierarchy between the weak scale and $\Lambda$. The argument from fits to the precision electroweak observables applies only if the standard model is the correct theory up to scales of at least $10 \mathrm{TeV}$; it is a very weak bound which is immediately evaded by large extra dimensions, allowing several scenarios for EWSB:

- Light Higgs $\left(m_{h}<200 \mathrm{GeV}\right)$ : For $\Lambda \gtrsim 20 \mathrm{TeV}$ some protection mechanism for the Higgs mass would be required; if this is supersymmetry, the Higgs will be light. For $\Lambda \approx 1-3 \mathrm{TeV}$, if the tree level Higgs mass happened to vanish, EWSB and a light Higgs boson could result from 1 loop radiative corrections.

- Heavy Higgs $\left(m_{h}>200 \mathrm{GeV}\right)$ : This could arise for $\Lambda \approx 1-3 \mathrm{TeV}$, if the Higgs mass parameter is somewhat less than $\Lambda$, or alternatively for $\Lambda \approx 3-10 \mathrm{TeV}$ if the Higgs mass parameter vanishes at tree level but arises at 1 loop. In both cases a large value for the Higgs self coupling is needed, and the operators (9) and (10) must mimic the effects of a light Higgs in the $S$ and $T$ parameters.

- No Higgs: Physics at the fundamental scale $\Lambda \approx 1-3 \mathrm{TeV}$ may itself cause EWSB. An example of this has already been proposed 11. In this case the theory below $\Lambda$ will have $S U(2) \times U(1)$ realized non-linearly, and the chiral Lagrangian will have 
operators analagous to (9) and (10) which mimic the effects of a light Higgs in the $S$ and $T$ parameters.

A light Higgs boson is just one possibility amongst several for EWSB, and is not preferred.

We have shown that, in theories with large extra dimensions having $\mathcal{O}_{B W, \Phi}$ with $c_{B W, \Phi}$ of order unity, the precision electroweak data provide a lower bound on the fundamental scale, $\Lambda_{\text {min }} \approx 3 \mathrm{TeV}$. For values of $\Lambda$ in the range $(1-3) \times \Lambda_{\text {min }}$, the signs $f_{B W, \Phi}$ are critical. For two sign choices, no successful fit can be found for any Higgs mass. For a third choice, a good fit to the data is found for Higgs masses all the way up to $m_{h}=800 \mathrm{GeV}$. For the final choice, masses up to $800 \mathrm{GeV}$ are also obtained, though the fits are less convincing. Only in the case of very large $\Lambda$ does the data still prefer a light Higgs, but then the quadratic finetuning of the light Higgs mass to one part in $m_{h}^{2} / \Lambda^{2}$ is reintroduced.

In view of the bounds on $\Lambda_{\text {min }}$ of $3-4 \mathrm{TeV}$ from each of $\mathcal{O}_{q q}(7), \mathcal{O}_{\ell q}(8), \mathcal{O}_{B W}(9)$, and $\mathcal{O}_{\Phi}(10)$, it may be felt that the exciting possibility of $\Lambda$ in the $1-3 \mathrm{TeV}$ range is unlikely. Why would all the relevant $c_{i}$ coefficients be small? One possibility is that the dominant interactions of the new physics at $\Lambda$ preserve symmetries that are broken by the electroweak gauge interactions, including $P, C P$ and custodial $S U(2)$. If these symmetries are broken by sub-dominant interactions at $\Lambda$, then the smallness of the relevant $c_{i}$ can be naturally explained.

\section{Higgs Production and Decay}

For the case that there is a Higgs boson, either light or heavy, we now study the effects of $\mathcal{O}_{G, \gamma}$ of (3)-(4) on the signal for $h \rightarrow \gamma \gamma$ at hadron colliders. These operators have two immediate consequences. First, when both Higgs fields are set to their vacuum expectation values (vev's), the gauge couplings of $\mathrm{QED}$ and QCD are shifted. But these shifts can be reabsorbed into the definition of the gauge couplings and therefore have no observable implications. (If one attempts to unify the SM gauge couplings at some ultraviolet scale, or otherwise define theoretical relations among them, then these shifts will enter into the relation between the theoretical couplings and those extracted from data. However, for all but the lightest $\Lambda$, this shift is smaller than the experimental uncertainties.)

The second consequence is the possibility of unusual production and decay modes of the (physical) Higgs bosons. Taking one of the Higgs fields to its vev, one obtains terms in the effective Lagrangian:

$$
\mathcal{L}_{e f f}=\cdots+f_{\gamma} \frac{v}{\Lambda_{\gamma}^{2}} h F_{\mu \nu} F^{\mu \nu}+f_{G} \frac{v}{\Lambda_{G}^{2}} h G_{\mu \nu}^{a} G^{a \mu \nu}+\cdots
$$

where $h$ is the physical Higgs boson, $v=246 \mathrm{GeV}$ and $f_{\gamma, g}= \pm 1$ are unknown signs. First, $\mathcal{O}_{G}$ can contribute to the gluon fusion process $g g \rightarrow h$. It is well-known that the dominant production mode for Higgs bosons at the Tevatron and the LHC is through gluon fusion, via a loop of $t$-quarks. Because the process occurs at one-loop, non-renormalizable operators are more likely to provide a significant correction to the cross-section. Integrating out the $t$-quark, the relevant low-energy operator is then (for a recent discussion of the relevant SM Higgs physics, see [12]):

$$
\mathcal{L}_{G, e f f}=\left(-\frac{g \alpha_{s}}{24 \pi M_{W}} I_{G}+f_{G} \frac{v}{\Lambda_{G}^{2}}\right) h G_{\mu \nu}^{a} G^{a \mu \nu}
$$


where $g$ is the $\mathrm{SU}(2)$ coupling constant and $I_{G} \rightarrow 1(0)$ for $m_{t}^{2} \gg m_{h}^{2}\left(m_{t}^{2} \ll m_{h}^{2}\right)$. For $\Lambda \lesssim 4.5 \mathrm{TeV}$, the new physics will actually dominate the production of Higgs bosons. Note that the cross-section is maximized for constructive interference, $f_{G}=-1$, and minimized for $f_{G}=+1$.

The operator $\mathcal{O}_{\gamma}$ does not contribute to Higgs production[?] However it can contribute to the decay of the Higgs into photons:

$$
\Gamma(h \rightarrow \gamma \gamma)=\frac{|\beta|^{2} m_{h}^{3}}{4 \pi}
$$

for $\mathcal{L}=\beta h F_{\mu \nu} F^{\mu \nu}$. In the SM, this process is dominated by loops of $W$-bosons and $t$-quarks. Integrating them out yields an effective operator:

$$
\mathcal{L}_{\gamma, e f f}=\left(-\frac{g \alpha}{4 \pi M_{W}} I_{\gamma}+f_{\gamma} \frac{v}{\Lambda_{\gamma}^{2}}\right) h F_{\mu \nu} F^{\mu \nu}
$$

where $I_{\gamma}$ varies from roughly -0.5 to -1.3 as $m_{h}$ is varied. Once again, the new physics will dominate the width for $h \rightarrow \gamma \gamma$ given $\Lambda_{\gamma} \lesssim 7 \mathrm{TeV}$. If $m_{h} \lesssim 150 \mathrm{GeV}$, its decay width is dominated by final state $b$-quarks; then $h \rightarrow \gamma \gamma$ becomes the dominant decay mode given $\Lambda_{\gamma} \lesssim 1.5 \mathrm{TeV}$. However, even for larger $\Lambda_{\gamma}$, the branching ratio $h \rightarrow \gamma \gamma$ may be more than sufficient to provide a strong signal. The signal is maximized for $f_{\gamma}=+1$ (i.e., constructive interference of the SM and new physics) and minimized for $f_{\gamma}=-1$.

(In the context of LEP, Ref. [13] recently examined the effect of $\mathcal{O}_{\gamma}$ and related operators on $e^{+} e^{-} \rightarrow 3 \gamma, q q \gamma \gamma$ and found sensitivity there to new physics roughly below a scale $\Lambda \lesssim 600 \mathrm{GeV}$.)

Unfortunately, the operator $\mathcal{O}_{G}$ can also contribute to the Higgs decay width via $h \rightarrow g g$ which is unobservable among the QCD backgrounds. In fact, to lowest order,

$$
\Gamma(h \rightarrow g g)=8\left(\frac{\Lambda_{\gamma}}{\Lambda_{G}}\right)^{4} \Gamma(h \rightarrow \gamma \gamma) .
$$

In the limit in which the new physics is dominating the Higgs decays and $\Lambda_{\gamma} \simeq \Lambda_{G}$, the $h \rightarrow g g$ decays suppress the branching ratio into $h \rightarrow \gamma \gamma$ by about a factor of 10 . However, once final state $W W / Z Z$ dominate the Higgs width, the decays to gluons provide no real additional suppression of the $h \rightarrow \gamma \gamma$ branching fraction. Finally we note that the interference of $\mathcal{O}_{G}$ with the SM gives simultaneously larger (smaller) Higgs cross-sections and larger (smaller) $\Gamma(h \rightarrow g g)$.

The sensitivity of any experiment to new physics in the Higgs channel is then a function of several variables: $m_{h}, f_{\gamma}, f_{G}, \Lambda_{\gamma}$ and $\Lambda_{G}$. There are four sign choices for $f_{\gamma}, f_{G}$; we choose to study the two cases which maximize/minimize the signal at current and future colliders. The maximum signal case has $f_{\gamma}=+1$ and $f_{G}=-1$; we checked that over the entire range of interest the increase in the cross-section implied by $f_{G}=-1$ more than offset the corresponding increase in $\operatorname{Br}(h \rightarrow g g)$. The minimum signal case has the opposite choice of both signs.

\footnotetext{
${ }^{2}$ However, a large coefficient to $\mathcal{O}_{\gamma}$ could turn the NLC into an $s$-channel Higgs factory when run in $\gamma \gamma$ mode.
} 
Our analysis then has two parts. First we ignore the $\mathcal{O}_{G}$ operator (i.e., $f_{G}=0$ ) and determine the sensitivity of current and future experiments to new physics through $\mathcal{O}_{\gamma}$ alone. In this case, the production cross-section is simply that of the SM. Then in a second analysis we include both $\mathcal{O}_{\gamma}$ and $\mathcal{O}_{G}$. As we already noted, the effect of $\mathcal{O}_{G}$ is both to enhance the production but also to diminish the relative branching ratio of $h \rightarrow \gamma \gamma$.

For the purposes of doing the numerical calculations, we have used (in a greatly modified form) the programs of M. Spira and collaborators [14]. In all cases, we will work only to leading order. In the SM it has been found that NLO QCD corrections can change the cross-sections and decay widths by $\sim 60 \%$ [12]. Naively such changes appear to correspond only to $\sim 10 \%$ shifts in $\Lambda$, which are too small for the physics we are interested in here. However, it is possible that interference effects and enhanced backgrounds (i.e., $h \rightarrow \gamma \gamma$ in the SM) could produce a larger effect — we will not consider that possibility here.

Throughout our analysis we also have to address issues of acceptances and backgrounds in an approximate manner. In Run I, CDF reported an efficiency times acceptance approaching $15 \%$ in inclusive $\gamma \gamma+X$ Higgs searches [15]; we will assume that this figure prevails at all future facilities. There are also two major sources of backgrounds for our $\gamma \gamma$ signal: SM processes which produce or fake $\gamma \gamma$, and the usual SM decay of $h \rightarrow \gamma \gamma$ itself. The latter can be calculated explicitly. For the former we estimate by fitting to the CDF background spectrum [15], appropriately scaled to the luminosity of future Tevatron runs, or the ATLAS background spectrum [16] appropriately scaled for LHC runs.

In Figures 2(a)-(b) we show the sensitivity to $\Lambda_{\gamma}$ that can be obtained at various machines by plotting their $5 \sigma$ discovery reaches (with no $\mathcal{O}_{G}$ contribution). The colliders shown are: the Tevatron with $\sqrt{s}=1.8 \mathrm{TeV}$ and $100 \mathrm{pb}^{-1}$ of luminosity (Run I), with $\sqrt{s}=2 \mathrm{TeV}$ and $2 \mathrm{fb}^{-1}$ of luminosity (Run II), with $\sqrt{s}=2 \mathrm{TeV}$ and $30 \mathrm{fb}^{-1}$ (a proposed Run III), and the LHC with $\sqrt{s}=14 \mathrm{TeV}$ and $10 \mathrm{fb}^{-1}$ (initial luminosity) and $100 \mathrm{fb}^{-1}$ (final luminosity) respectively. (Note that the TeV Run I line falls below the region of parameter space plotted.) As one expects, once the $h \rightarrow W W, Z Z$ threshold opens up at $\sqrt{s} \simeq 150 \mathrm{GeV}$, the large $\Gamma(h \rightarrow W W, Z Z)$ is sufficient to overwhelm the photonic width and our experimental sensitivity drops significantly. Nonetheless, given the possibility of a light Higgs (and the robust arguments for one in supersymmetric frameworks) experimentalists should be encouraged to view $h \rightarrow \gamma \gamma$ as a viable and potentially large signal.

In terms of extracting a conservative discovery reach for $\Lambda$, Figure 2(b) should be used since it chooses $f_{\gamma}$ in order to minimize the signal. We note, for example, that the data from Run I cannot presently probe (or exclude) $\Lambda$ above $1 \mathrm{TeV}$, but that Run II should have a reach of approximately $1-1.5 \mathrm{TeV}$ for a light Higgs. However it is important to realize that for generic $f_{\gamma}$, the various colliders may have reaches as high as those shown in Figure 2(a). Thus, for example, if the Higgs mass is below the $W W$ threshold, the LHC can possibly find a signal for $\Lambda$ up to $8 \mathrm{TeV}$ for a light Higgs! (Unfortunately, that scale could also be as low as $4 \mathrm{TeV}$.)

Figures $3(\mathrm{a})$-(b) repeat the same analysis, but now with $\mathcal{O}_{G}$ included such that $\Lambda_{G}=$ $\Lambda_{\gamma} \equiv \Lambda$. We view these results as more realistic compared to those above in which only the $\mathcal{O}_{\gamma}$ operator was kept. We again show the same set of 5 collider options. Figure $3(\mathrm{~b})$ is the conservative $5 \sigma$ discovery reach, chosen to minimize the $p p, p \bar{p} \rightarrow h \rightarrow \gamma \gamma$ rate. It is interesting that for a light Higgs, the limits are slightly stronger than those obtained with $f_{G}=0$; now even the Tevatron Run I data has the ability to probe scales above $1 \mathrm{TeV}$. 

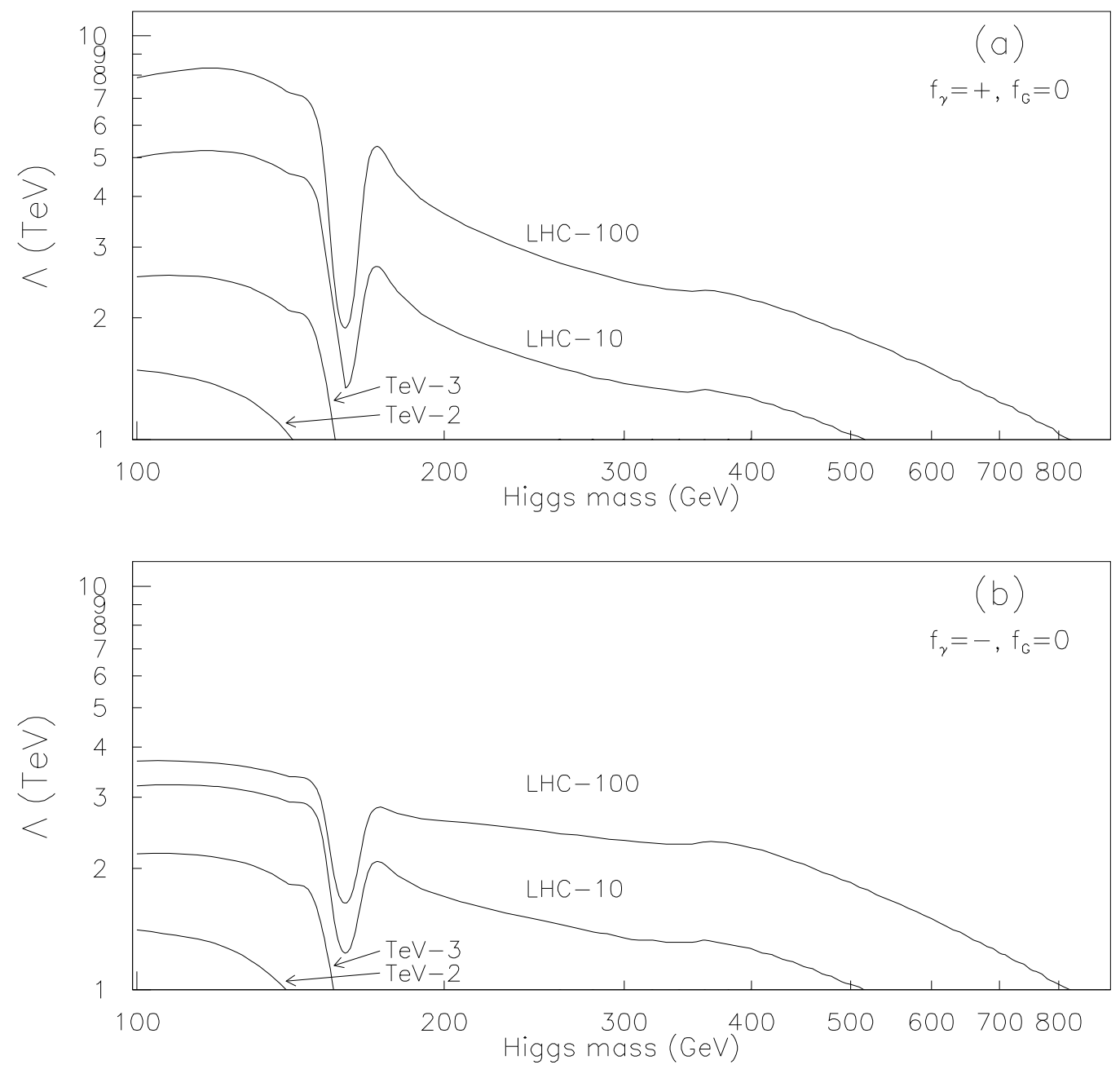

Figure 2: $5 \sigma$ discovery reaches for $p p, p \bar{p} \rightarrow h \rightarrow \gamma \gamma$ in current and future colliders. Only the $\mathcal{O}_{\gamma}$ operator has been included. In (a), signs are chosen to maximize the signal, while they are chosen to minimize the signal in (b). 


\begin{tabular}{l|cc|cc|cc}
\multicolumn{7}{c|}{$m_{h}(\mathrm{GeV})$} \\
\multicolumn{1}{c|}{$\Lambda(\mathrm{TeV})$} & \multicolumn{2}{|c}{110} & \multicolumn{2}{c}{200} & \multicolumn{2}{c}{500} \\
\hline Tev Run I & 2.0 & 1.8 & 1.1 & - & - & - \\
Tev Run II & 2.6 & 3.0 & 1.5 & 1.3 & - & - \\
Tev Run III & 3.0 & 4.2 & 1.8 & 1.8 & 1.1 & - \\
LHC $\left(10 \mathrm{fb}^{-1}\right)$ & 3.4 & 7.2 & 2.9 & 3.5 & 2.3 & 2.1 \\
LHC $\left(100 \mathrm{fb}^{-1}\right)$ & 3.5 & 10.8 & 3.2 & 5.8 & 2.9 & 2.9
\end{tabular}

Table 1: Exclusion limits and maximum discovery reaches (in $\mathrm{TeV}$ ) for various collider runs for 3 representative Higgs masses. The first column for each $m_{h}$ is a conservative $2 \sigma$ exclusion reach for each machine; the second column is the optimistic $5 \sigma$ discovery reach. Unfilled columns represent limits below $1 \mathrm{TeV}$. We take $\Lambda_{G}=\Lambda_{\gamma}$ for the table.

However the more noticable difference is the ability to produce larger numbers of heavy Higgs bosons and observe their $\gamma \gamma$ decays. For example, the LHC is capable of probing scales near $2 \mathrm{TeV}$ even for $m_{h}=1 \mathrm{TeV}$.

Figure 3(b) shows the maximal reach of the various colliders, with the LHC now extending its sensitivity to $\Lambda$ as high as $10 \mathrm{TeV}$ for a light Higgs! Finally, we summarize a few of our results for $m_{h}=110,200$ and $500 \mathrm{GeV}$ for both exclusion and discovery in Table 2. All bounds assume $\Lambda_{\gamma}=\Lambda_{G}$. For each choice of the Higgs mass, we have shown a conservative limit on $\Lambda$ which can be excluded, and a maximum $\Lambda$ below which a signal may be discovered. Thus for the exclusion bounds $(2 \sigma)$ we have taken the interference effects to minimize the signal; for the maximum discovery reaches $(5 \sigma)$, we have chosen the interference effects to maximize the signal.

We have attempted in this analysis to be rather conservative. For one thing, the $2 \sigma$ exclusion limits of the various colliders are often several $\mathrm{TeV}$ higher than the $5 \sigma$ discovery limits. Secondly, we have treated the discovery of the $h \rightarrow \gamma \gamma$ signal as simply a counting experiment, throwing away useful experimental information, for example on the shape of the diphoton mass spectrum, which would be available experimentally to help extract the signal from the backgrounds. Lastly, we have not included QCD corrections to the amplitudes, which we believe could increase the signal (though also increasing the "background" $h \rightarrow \gamma \gamma$ signal) by $\sim 50 \%$. Therefore we believe that the reaches given here are to be taken as conservative values, insofar as one should take the scales deduced from naive power-counting seriously.

\section{Conclusions}

In this paper we have studied two consequences of large extra dimensions for electroweak symmetry breaking: a relaxation of the precision electroweak bound on the Higgs boson mass, and an enhanced rate for $\gamma \gamma$ events at hadron colliders from Higgs decay.

The relaxation of the precision electroweak bound on the Higgs mass applies when any new physics generates (9) and (10) at a scale of several TeV. It is well known that $S$ and $T$ depend only logarithmically on the Higgs boson mass, but it may not be appreciated that the mass bound can be evaded completely for a wide range of values of $\Lambda$, extending as high as $10 \mathrm{TeV}$. For example, even a weakened bound of $m_{h}<500 \mathrm{GeV}$, only applies if the 

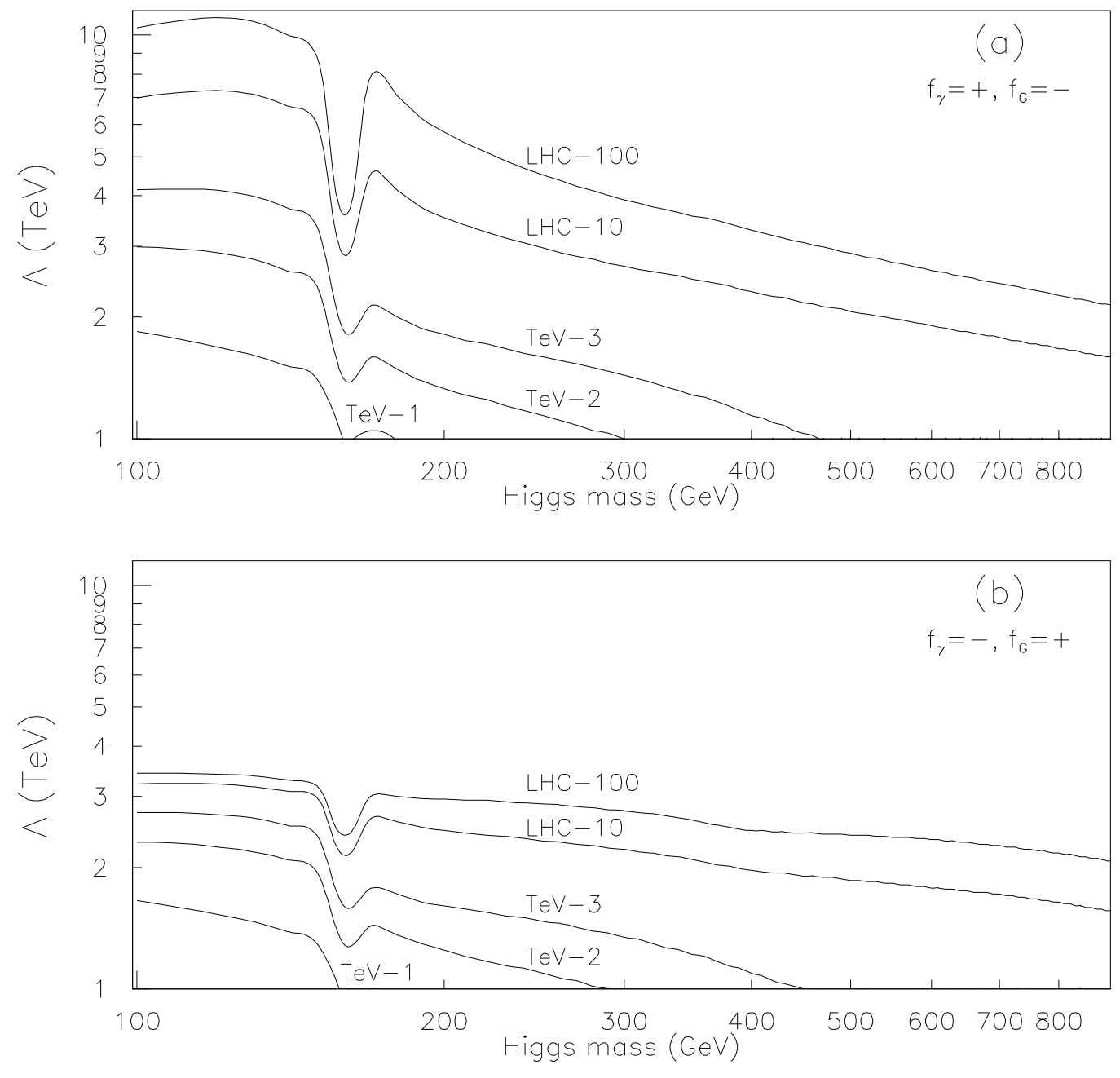

Figure 3: $5 \sigma$ discovery reaches for $p p, p \bar{p} \rightarrow h \rightarrow \gamma \gamma$ in current and future colliders. Both $\mathcal{O}_{G}$ and $\mathcal{O}_{\gamma}$ have been included, with $\Lambda_{\gamma}=\Lambda_{G} \equiv \Lambda$. In (a), signs are chosen to maximize the signal, while they are chosen to minimize the signal in (b). 
standard model is the correct description of nature up to energies of $17 \mathrm{TeV}$. We find this implausible, since it implies a fine tuning in the Higgs mass squared parameter of 1 part in 2000. There is only one strong argument for a light Higgs boson: the correct successful prediction of the weak mixing angle at the percent level of accuracy requires weak scale supersymmetry, and therefore a light Higgs boson. In theories with large extra dimensions this argument is not applicable, since the percent level prediction for the weak mixing angle is lost. Hence, in these theories, there is no preference for a light Higgs boson, and thus alternatives with a heavy Higgs or no Higgs should be considered seriously.

If there is a Higgs boson, we have shown that a generic signal of large extra dimensions is an anomalously large $\gamma \gamma$ signal at machines capable of producing Higgs bosons. Expectations from the SM put such a signal out of reach of the Tevatron. In Figure 3 we showed the $5 \sigma$ discovery reaches for $h \rightarrow \gamma \gamma$ at the Tevatron and LHC. At Run II of the Tevatron collider this signal would be discovered for a light Higgs if $\Lambda$ is less than 2 (3) TeV for destructive (constructive) interference. LHC not only increases the discovery potential for a light Higgs boson mass, up to $10 \mathrm{TeV}$ for constructive interference, but also has significant discovery potential up to the largest Higgs masses. This signal compares favorably with that of graviton production at colliders [17], especially if the scale which sets the size of the $4+n$ dimensional gravitational coupling is somewhat larger than the scale $\Lambda$.

\section{Acknowledgements}

We are grateful to Nima Arkani-Hamed, Michael Chanowitz, Savas Dimopoulos and Henry Frisch for many useful conversations. This work was supported in part by the U.S. Department of Energy under contract DE-AC03-76SF00098 and by the National Science Foundation under grant PHY-95-14797.

\section{References}

[1] N. Arkani-Hamed, S. Dimopoulos and G. Dvali, Phys. Lett. B429 (1998) 263 and Phys. Rev. D59 (1999) 086004;

N. Arkani-Hamed, S. Dimopoulos and J. March-Russell, hep-th/9809124.

[2] T. Banks, M. Dine and A. Nelson, hep-th/9903019.

[3] C. Arzt, M. Einhorn and J. Wudka, Nucl. Phys. B433 (1995) 41.

[4] K. Hagiwara, R. Szalapski and D. Zeppenfeld, Phys. Lett. B318 (1993) 155; M. Gonzalez-Garcia, S. Lietti and S. Novaes, Phys. Rev. D57 (1998) 7045.

[5] A. Deandrea, Phys. Lett. B409 (1997) 277.

[6] K. Ackerstaff et al. (OPAL Collaboration), Phys. Lett. B391 (1997) 221. An unpublished result presented by R. Clare at the LEPC meeting of November 1998 is only slightly stronger.

[7] F. Abe et al. (CDF Collaboration), Phys. Rev. Lett. 79 (1997) 2198. 
[8] K. Hagiwara, T. Hatsukano, S. Ishihara and R. Szalapski, Nucl. Phys. B496 (1997) 66 ;

K. Hagiwara, D. Haidt and S. Matsumoto, Eur. Phys. J. C2 (1997) 95.

[9] J. Erler, hep-ph/9903449.

[10] G. Kane, C. Kolda and J. Wells, Phys. Rev. Lett. 70 (1993) 2686;

J. Espinosa and M. Quiros, Phys. Lett. B302 (1993) 51.

[11] N. Arkani-Hamed and S. Dimopoulos, hep-ph/9811353.

[12] M. Spira, A. Djouadi, D. Graudenz and P. Zerwas, Nucl. Phys. B453 (1995) 17.

[13] O. Éboli, M. Gonzalez-Garcia, S. Lietti and S. Novaes, Phys. Lett. B434 (1998) 340.

[14] M. Spira, hep-ph/9510347;

A. Djouadi, J. Kalinowski and M. Spira, hep-ph/9704448.

[15] P. Wilson (CDF Collaboration), "Search for High Mass Photon Pairs in $p \bar{p}$ Collisions at $\sqrt{s}=1.8 \mathrm{TeV}$," contributed to ICHEP 98, preprint FERMILAB-CONF-98/213-E (Jun 1998).

[16] W. Armstrong et al. (ATLAS Collaboration), "ATLAS Technical Proposal," preprint CERN/LHCC/94-43 (Dec 1994).

[17] G. Giudice, R. Rattazzi and J. Wells, hep-ph/9811291;

E. Mirabelli, M. Perelstein and M. Peskin, hep-ph/9811337. 